\title{
ON THE SOLUTIONS OF A CLASS OF LINEAR SELFADJOINT DIFFERENTIAL EQUATIONS
}

\author{
BY \\ LARRY R. ANDERSON( $\left.{ }^{1}\right)$ AND A. C. LAZER( $\left.{ }^{2}\right)$
}

\begin{abstract}
Let $L$ be a linear selfadjoint ordinary differential operator with coefficients which are real and sufficiently regular on $(-\infty, \infty)$. Let $A^{+}\left(A^{-}\right)$denote the subspace of the solution space of $L y=0$ such that $y \in A^{+}\left(y \in A^{-}\right)$iff $D^{k} y \in L^{2}[0, \infty)$ $\left(D^{k} y \in L^{2}(-\infty, 0]\right)$ for $k=0,1, \ldots, m$ where $2 m$ is the order of $L$. A sufficient condition is given for the solution space of $L y=0$ to be the direct sum of $A^{+}$and $A^{-}$. This condition which concerns the coefficients of $L$ reduces to a necessary and sufficient condition when these coefficients are constant. In the case of periodic coefficients this condition implies the existence of an exponential dichotomy of the solution space of $L y=0$.
\end{abstract}

1. Introduction. The object of study of this paper is the general linear homogeneous selfadjoint differential equation which for convenience we shall write in the form

$$
\sum_{k=0}^{m}(-1)^{k} D^{k} a_{k} D^{k} y=0
$$

where $D^{k} y \equiv d^{k} y / d t^{k}$.

Except when otherwise stated we will assume throughout that for each $k=0$, $1, \ldots, m, a_{k}(t)$ is real valued, $a_{k} \in C^{k}(-\infty, \infty)$ and $a_{m}(t) \neq 0$ for all $t \in(-\infty, \infty)$.

The motivation for this paper comes from the case when $a_{k}(t)=c_{k}=$ constant, $k=0,1, \ldots, m$. In this case the solutions of (1) are determined entirely by the zeros of the polynomial

$$
p(\lambda)=\sum_{k=0}^{m}(-1)^{k} c_{k} \lambda^{2 k} .
$$

Since only even powers of $\lambda$ appear in $p$ it follows that if $\mu \neq 0$ is a zero of $p$ of multiplicity $r$ then $-\mu$ is also a zero of $p$ of multiplicity $r$ and the functions $t^{j} e^{\mu t}$, $t^{j} e^{-\mu t}, j=0,1, \ldots, r-1$, form a set of $2 r$ linearly independent solutions of (1). Consequently if $\bar{p}(\lambda)$ has no zero or purely imaginary roots and $S$ denotes the set of solutions of (1) considered as a complex vector space of dimension $2 m$, then $S$ has a simple geometrical description. Namely, if $E^{+}$denotes the subspace of $S$ consisting

Received by the editors August 27, 1969.

AMS subject classifications. Primary 3420, 3422.

Key words and phrases. $L^{2}$ solutions of linear differential equations, periodic coefficients, asymptotic behavior of solutions.

( $\left.{ }^{1}\right)$ Partially supported by NASA under grant no. NS G(T)-42.

( $\left.{ }^{2}\right)$ Partially supported by NSF under grant no. NSF GP-8961.

Copyright (C) 1970, American Mathematical Society 
of solutions of (1) which together with their derivatives tend to zero exponentially as $t \rightarrow \infty$ and $E^{-}$denotes the subspace of $S$ consisting of solutions of (1) which together with their derivatives tend to zero as $t \rightarrow-\infty$ exponentially then dimension $E^{+}=$dimension $E^{-}=m$, dimension $E^{+} \cap E^{-}=0$. Therefore $S$ will split into the direct sum of $E^{+}$and $E^{-}$.

The objective of this paper is to give a partial extension of this simple observation to a class of equations of the form (1) with variable coefficients. For simplicity we will only consider real solutions. Henceforth $S$ will denote the set of real solutions of (1) considered as a real vector space of dimension $2 \mathrm{~m}$.

THEOREM 1. Assume that for each $k=0,1, \ldots, m, a_{k}(t)$ is bounded below on $(-\infty, \infty)$ and define

$$
c_{k}=\inf a_{k}(t) .
$$

Let $A^{+}$and $A^{-}$denote the subspaces of $S$ defined by

$$
\begin{aligned}
& A^{+}=\left\{v \in S \mid \begin{array}{l}
D^{k} v \in L^{2}[0, \infty) \\
0 \leqq k \leqq m
\end{array}\right\} \\
& A^{-}=\left\{v \in S \mid \begin{array}{l}
D^{k} v \in L^{2}(-\infty, 0] \\
0 \leqq k \leqq m
\end{array}\right\}
\end{aligned}
$$

If

$$
c_{m}>0
$$

and the polynomial p defined by (2) has no zero or purely imaginary roots then

$$
\operatorname{dim} A^{+} \geqq m, \quad \operatorname{dim} A^{-} \geqq m .
$$

If, in addition, each $a_{k}(t)$ is bounded above as well as below then

$$
\text { dimension } A^{+}=\text {dimension } A^{-}=m
$$

and

$$
\text { dimension } A^{+} \cap A^{-}=0
$$

so that $S$ is the direct sum of $A^{+}$and $A^{-}$.

If $v \in A^{+}\left(v \in A^{-}\right)$then

$$
\lim _{t \rightarrow \infty} D^{k} v=0 \quad\left(\lim _{t \rightarrow-\infty} D^{k} v=0\right), \quad k=0,1, \ldots, m-1 .
$$

To the best of our knowledge the only literature connected with Theorem 1 is a remarkable paper by $M$. Švec [3] which deals with the fourth order equation $d^{4} y / d t^{4}+p(t) y=0$ where $p$ is defined and continuous on a half-infinite interval $[c, \infty)$. Švec showed that if $p$ is bounded below by a positive constant then there exist two linearly independent solutions of the differential equation which belong to $L^{2}[c, \infty)$ and tend to zero as $t \rightarrow \infty$. As an application of Theorem 2, which 
is similar to Theorem 1 but concerns the differential equation (1) when the $a_{k}$ are only defined on a half-infinite interval $[c, \infty)$, we shall generalize Švec's result.

The proof of Theorem 1 will be deferred until after we have established some auxiliary lemmas.

\section{Some preliminary lemmas.}

LeMmA 2.1. Let $d_{k}, k=0,1, \ldots, m$, be real numbers with the property that

$$
q(\omega)=\sum_{k=0}^{m} d_{k} \omega^{2 k} \geqq 0
$$

for all real $\omega$. Let $f$ be a real function of class $C^{m-1}$ on $[-T, T], T>0$, and sectionally of class $C^{m}$ on this interval, i.e. there exist numbers $t_{j}, j=1, \ldots, N-1$, such that

$$
-T=t_{0}<t_{1}<\cdots<t_{N-1}<t_{N}=T
$$

and $f$ is of class $C^{m}$ on each of the intervals $\left[t_{j-1}, t_{j}\right], j=1, \ldots, N$. If

$$
D^{k} f(-T)=D^{k} f(T)=0, \quad k=0,1, \ldots, m-1,
$$

then

$$
\int_{-T}^{T} \sum_{k=0}^{m} d_{k}\left(D^{k} f(s)\right)^{2} d s \geqq 0
$$

Proof. If for $t \in[-\pi, \pi]$ we define $F(t)=f(t T / \pi)$ then $F$ is of class $C^{m-1}$ on $[-\pi, \pi], F$ is sectionally of class $C^{m}$ on this interval,

$$
D^{k} F(-\pi)=D^{k} F(\pi)=0, \quad 0 \leqq k \leqq m-1,
$$

and

8)

$$
\int_{-T}^{T} \sum_{k=0}^{m} d_{k}\left(D^{k} f(s)\right)^{2} d s=\frac{1}{r} \int_{-\pi}^{\pi} \sum_{k=0}^{m} d_{k} r^{2 k}\left(D^{k} F(u)\right)^{2} d u,
$$

where $r=\pi / T$.

For each $j=0, \pm 1, \pm 2, \ldots$ let

$$
\gamma_{j}=\frac{1}{(2 \pi)^{1 / 2}} \int_{-\pi}^{\pi} F(u) e^{-i j u} d u
$$

Integration by parts and (7) yield

$$
(-i j)^{k} \gamma_{j}=\frac{1}{(2 \pi)^{1 / 2}} \int_{-\pi}^{\pi} D^{k} F(u) e^{-i j u} d u,
$$

for $k=1, \ldots, m-1$. Since $D^{m} F$ is sectionally continuous it follows by dividing the interval of integration in (9) into suitable subintervals that (9) is also true for $k=m$.

The orthonormal functions

$$
\left(1 /(2 \pi)^{1 / 2}\right) e^{i j u}, \quad j=0, \pm 1, \pm 2, \ldots,
$$


form a complete set in $L^{2}[-\pi, \pi]$, so by Parseval's formula

$$
\int_{-\pi}^{\pi}\left(D^{k} F(u)\right)^{2} d u=\sum_{j=-\infty}^{\infty} j^{2 k}\left|\gamma_{j}\right|^{2}
$$

for $k=0,1, \ldots, m\left(0^{\circ} \equiv 1\right.$ in the above and following identity). Hence

$$
\begin{aligned}
\int_{-\pi}^{\pi} \sum_{k=0}^{m} d_{k} r^{2 k}\left(D^{k} F(u)\right)^{2} d u & =\sum_{k=0}^{m} d_{k} r^{2 k} \sum_{j=-\infty}^{\infty} j^{2 k}\left|\gamma_{j}\right|^{2} \\
& =\sum_{j=-\infty}^{\infty}\left|\gamma_{j}\right|^{2} \sum_{k=0}^{m} d_{k}(j r)^{2 k}=\sum_{j=-\infty}^{\infty}\left|\gamma_{j}\right|^{2} q(r j) \geqq 0 .
\end{aligned}
$$

By (8), this proves the lemma.

LEMMA 2.2. Let the real numbers $d_{0}, d_{1}, \ldots, d_{m}$ satisfy the same hypothesis as in Lemma 2.1. Let $f$ be a real valued function defined and of class $C^{m}$ on the interval $[0, T], T>0$. If

$$
D^{k} f(T)=0, \quad 0 \leqq k \leqq m-1,
$$

and for some fixed integer $j$ with $0 \leqq j \leqq m-1$,

$$
D^{k} f(0)=0, \quad k \neq j, 0 \leqq k \leqq m-1,
$$

then

$$
\sum_{k=0}^{m} \int_{0}^{T} d_{k}\left(D^{k} f(s)\right)^{2} d s \geqq 0 .
$$

Proof. We define a function $g$ on $[-T, T]$ as follows:

If $j$ is an even integer

If $j$ is an odd integer

$$
\begin{aligned}
g(t) & =f(t), & & 0 \leqq t \leqq T, \\
& =f(-t), & & -T \leqq t<0 .
\end{aligned}
$$

$$
\begin{aligned}
g(t) & =f(t), & & 0 \leqq t \leqq T, \\
& =-f(-t), & & -T \leqq t<0 .
\end{aligned}
$$

Using (11) it is easy to verify that $g$ is of class $C^{m-1}$ on $[-T, T]$ and sectionally of class $C^{m}$ on this interval since $D^{m} g$ has both left-hand and right-hand limits at $t=0$. From $(10) D^{k} g(-T)=D^{k} g(T)=0,0 \leqq k \leqq m-1$. Thus Lemma 1.1 is applicable and

$$
\int_{-T}^{T} \sum_{k=0}^{m} d_{k}\left(D^{k} g(s)\right)^{2} d s \geqq 0
$$

But

$$
\int_{0}^{T} \sum_{k=0}^{m} d_{k}\left(D^{k} f(s)\right)^{2} d s=\frac{1}{2} \int_{-T}^{T} \sum_{k=0}^{m} d_{k}\left(D^{k} g(s)\right)^{2} d s
$$

and the assertion of the lemma follows. 
LEMMA 2.3. Let the real numbers $d_{0}, d_{1}, \ldots, d_{m}$ satisfy the same hypothesis as in Lemma 2.1. If $f \in C^{m}(-\infty, \infty)$ and $D^{k} f \in L^{2}(-\infty, \infty), k=0,1, \ldots, m$, then

$$
\int_{-\infty}^{\infty} \sum_{k=0}^{m} d_{k}\left(D^{k} f(s)\right)^{2} d s \geqq 0 .
$$

Proof. This result is almost an immediate consequence of Lemma 2.1. Let $\varphi(t)$ be a real valued function defined and of class $C^{\infty}$ on the real line such that $\varphi(t)=1$ for $t \leqq \frac{1}{2}$ and $\varphi(t)=0$ for $t \geqq 1$. For each positive integer $n$ let $\theta_{n}$ be the $C^{\infty}$ function defined by

$$
\begin{aligned}
\theta_{n}(t) & =1, & & 0 \leqq t \leqq n, \\
& =\varphi(t-n), & & n<t, \\
& =\theta_{n}(-t), & & t<0 .
\end{aligned}
$$

Let $f_{n}=\theta_{n} f$ for $n=1,2, \ldots$ Since $D^{k} \theta_{n}$ is bounded independently of $n$ for $0 \leqq k \leqq m$ there exists a fixed constant $L$ such that

$$
\left(D^{k} f_{n}\right)^{2} \leqq L \sum_{j=0}^{m}\left(D^{j} f\right)^{2}
$$

for $k$ and $n$ in the same range. For each fixed $t \in(-\infty, \infty), \lim _{n \rightarrow \infty} D^{k} f_{n}(t)=D^{k} f(t)$ so by the dominated convergence theorem

$$
\int_{-\infty}^{\infty} \sum_{k=0}^{m} d_{k}\left(D^{k} f(s)\right)^{2} d s=\lim _{n \rightarrow \infty} \int_{-\infty}^{\infty} \sum_{k=0}^{n} d_{k}\left(D^{k} f_{n}(s)\right)^{2} d s
$$

Since for each $n, f_{n}$ has compact support, it follows from Lemma 2.1 that

$$
\int_{-\infty}^{\infty} \sum_{k=0}^{n} d_{k}\left(D^{k} f_{n}(s)\right)^{2} d s \geqq 0
$$

This proves the lemma.

3. Proof of Theorem 1. In addition to the preliminary lemmas the proof of Theorem 1 will depend on a certain identity which we first establish.

For each solution $y$ of (1) we define a function $F[y]$ on $(-\infty, \infty)$ by the formula

$$
F[y](t)=\sum_{k=1}^{m} \sum_{j=0}^{k-1}(-1)^{j+k}\left(D^{j} y\right)(t)\left(D^{k-j-1} a_{k} D^{k} y\right)(t) .
$$

According to (1)

$$
\int_{0}^{t} y(s) \sum_{k=0}^{m}(-1)^{k}\left(D^{k} a_{k} D^{k} y\right)(s) d s=0
$$

so by the integration by parts formula

$$
\left.\int_{0}^{t} y D^{k} z d s=\sum_{j=0}^{k-1}(-1)^{j}\left(D^{j} y\right)\left(D^{k-j-1} z\right)\right]_{0}^{t}+(-1)^{k} \int_{0}^{t} z D^{k} y d s,
$$


we obtain the important identity

$$
F[y](t)=F[y](0)-\sum_{k=0}^{m} \int_{0}^{t} a_{k}(s)\left(D_{k} y(s)\right)^{2} d s .
$$

The proof of Theorem 1 will be broken up into several lemmas.

LeMmA 3.1. Let the coefficients $a_{k}(t)$ be bounded below on $(-\infty, \infty)$ and assume that the numbers $c_{k}$ satisfy the hypothesis of Theorem 1. Let $v$ be a solution of (1) such that for some number $T>0$,

$$
D^{k} v(T)=0, \quad 0 \leqq k \leqq m-1,
$$

and for some fixed integer $j$ with $0 \leqq j \leqq m-1$,

$$
D^{k} v(0)=0, \quad k \neq j, 0 \leqq k \leqq m-1 .
$$

There exists a number $M>0$ independent of both $v$ and $T$, such that

$$
\sum_{k=0}^{m} \int_{0}^{T}\left(D^{k} v(s)\right)^{2} d s \leqq M F[v](0)
$$

Proof. Since the polynomial $p(\lambda)=\sum_{k=0}^{m}(-1)^{k} c_{k} \lambda^{2 k}$ has no zero or purely imaginary roots it follows that if $Q(\omega) \equiv p(i \omega)=\sum_{k=0}^{m} c_{k} \omega^{2 k}$ then $Q(\omega) \neq 0$ for all $\omega \in(-\infty, \infty)$. According to assumption (6) $c_{m}>0$ and hence

$$
\lim _{\omega \rightarrow \pm \infty} Q(\omega)=+\infty \text {. }
$$

Thus $Q(\omega)>0$ for all real $\omega$ and in particular $Q(0)=c_{0}>0$. This together with (17) implies the existence of a number $\delta>0$ such that if

$$
d_{k} \equiv c_{k}-\delta, \quad 0 \leqq k \leqq m,
$$

then

$$
q(\omega) \equiv \sum_{k=0}^{m} d_{k} \omega^{2 k} \geqq 0, \quad \omega \in(-\infty, \infty) .
$$

Now by (14) and (12) it follows that $F[v](T)=0$ and so by (13)

$$
F[v](0)=\sum_{k=0}^{m} \int_{0}^{T} a_{k}(s)\left(D^{k} v(s)\right)^{2} d s .
$$

From (2) $a_{k}(t) \geqq c_{k}, 0 \leqq k \leqq m$, so by using (18) we have

$$
F[v](0) \geqq \sum_{k=0}^{m} \int_{0}^{T} c_{k}\left(D^{k} v(s)\right)^{2} d s=\sum_{k=0}^{m} \int_{0}^{T} d_{k}\left(D^{k} v(s)\right)^{2} d s+\delta \sum_{k=0}^{m} \int_{0}^{T}\left(D^{k} v(s)\right)^{2} d s .
$$

From (14), (15) and (19) we observe that the function $v$ and the numbers $d_{k}$ satisfy the hypothesis of Lemma 2.2 and hence

$$
\sum_{k=0}^{m} \int_{0}^{T} d_{k}\left(D^{k} v(s)\right)^{2} d s \geqq 0
$$


The assertion of the lemma follows by setting $M=1 / \delta$.

Lemma 3.2. Let the hypothesis of Lemma 3.1 hold. For each integer $j$ with $0 \leqq j \leqq m-1$ there exists a solution $v_{j}$ of (1) such that

$$
\begin{array}{ll}
D^{k} v_{j} \in L^{2}[0, \infty), & 0 \leqq k \leqq m, \\
D^{k} v_{j}(0)=0, & k \neq j, 0 \leqq k \leqq m-1,
\end{array}
$$

and

$$
D^{j} v_{j}(0) \neq 0 .
$$

Proof. Let $z_{i}, 0 \leqq i \leqq 2 m-1$, denote the solution of (1) defined by the initial conditions

$$
\begin{aligned}
D^{k} z_{i}(0)=\delta_{i k} & =0, & & i \neq k, \\
& =1, & & i=k .
\end{aligned}
$$

The solutions $z_{0}, z_{1}, \ldots, z_{2 m-1}$ obviously form a basis for the vector space $S$.

Let $0 \leqq j \leqq m-1$. By a well-known result of algebra, for each positive integer $n$ there exist $m+1$ numbers, which we denote by $b_{n}^{j}, b_{n}^{m}, b_{n}^{m+1}, \ldots, b_{n}^{2 m-1}$, not all zero such that

$$
b_{n}^{j} D^{k} z_{j}(n)+\sum_{i=m}^{2 m-1} b_{n}^{i} D^{k} z_{i}(n)=0 \quad \text { for } k=0,1, \ldots, m-1 .
$$

By a suitable normalization we may further assume that for all $n=0,1,2, \ldots$,

$$
\left(b_{n}^{j}\right)^{2}+\sum_{i=m}^{2 m-1}\left(b_{n}^{i}\right)^{2}=1 .
$$

For each positive integer $n$ consider the solution

$$
v_{j n}=b_{n}^{j} z_{j}+\sum_{i=m}^{2 m-1} b_{n}^{i} z_{i}
$$

From (22) and (23) $D^{k} v_{j n}(0)=0, k \neq j, 0 \leqq k \leqq m-1, D^{k} v_{j n}(n)=0,0 \leqq k \leqq m-1$. Thus if $M$ is defined as in Lemma 3.1, it follows that for all $n=0,1,2, \ldots$,

$$
\sum_{k=0}^{m} \int_{0}^{n}\left(D^{k} v_{j n}(s)\right)^{2} d s \leqq M F\left[v_{j n}\right](0) .
$$

Condition (24) implies the existence of a sequence of integers $\left\{n_{h}\right\}$ and $m+1$ numbers $b^{j}, b^{m}, b^{m+1}, \ldots, b^{2 m-1}$ such that $\lim _{h \rightarrow \infty} b_{n_{h}}^{i}=b^{i}, i=j, m \leqq i \leqq 2 m-1$, and

$$
\left(b^{j}\right)^{2}+\sum_{i=m}^{2 m-1}\left(b^{i}\right)^{2}=1 .
$$

We will show that the solution

$$
v_{j}=b^{j} z_{j}+\sum_{i=m}^{2 m-1} b^{i} z_{i}
$$

fulfills the assertion of the lemma. 
Fix $t>0$. Since by (25) the sequences $\left\{D^{k} v_{j n_{h}}\right\}$ converges uniformly to $D^{k} v_{j}$, $0 \leqq k \leqq m$, on bounded intervals

$$
\sum_{k=0}^{m} \int_{0}^{t}\left(D^{k} v_{j}(s)\right)^{2} d s=\lim _{h \rightarrow \infty} \sum_{k=0}^{m} \int_{0}^{t}\left(D^{k} v_{j n_{h}}(s)\right)^{2} d s
$$

For $n_{h} \geqq t$ it follows by (26) that

$$
\sum_{k=0}^{m} \int_{0}^{t}\left(D^{k} v_{j n_{h}}(s)\right)^{2} d s \leqq \sum_{k=0}^{m} \int_{0}^{n_{h}}\left(D^{k} v_{j n_{h}}(s)\right)^{2} d s \leqq M F\left[v_{j n_{h}}\right](0) .
$$

From (12), (25), and (28) we see that

Hence

$$
\lim _{h \rightarrow \infty} F\left[v_{j n_{h}}\right](0)=F\left[v_{j}\right](0) .
$$

$$
\sum_{k=0}^{m} \int_{0}^{t}\left(D^{k} v_{j}(s)\right)^{2} d s \leqq M F\left[v_{j}\right](0) .
$$

Since $t>0$ was arbitrary this implies that $D^{k} v_{j} \in L^{2}[0, \infty)$ for $0 \leqq k \leqq m$ and

$$
\sum_{k=0}^{m} \int_{0}^{\infty}\left(D^{k} v_{j}(s)\right)^{2} d s \leqq M F\left[v_{j}\right](0) .
$$

Finally, suppose contrary to the lemma $D^{j} v_{j}(0)=0$. By (22) and (28) $D^{k} v_{j}(0)=0$, $0 \leqq k \leqq m-1$, so by (12) $F\left[v_{j}(0)\right]=0$. Hence

$$
\sum_{k=0}^{m} \int_{0}^{\infty}\left(D^{k} v_{j}(s)\right)^{2} d s=0
$$

and $v_{j}(t)=0$ for all $t$. This, however, contradicts (27), (28) and the linear independence of the solutions $z_{j}, z_{m}, z_{m+1}, \ldots, z_{2 m-1}$. Hence $D^{j} v_{j}(0) \neq 0$ and the lemma is proved.

From this lemma the first assertion of Theorem 1 follows immediately. For each $j$ with $0 \leqq j \leqq m-1$, let $v_{j}$ be the solution whose existence was established above. If $v_{0}, v_{1}, v_{m-1}$ were not linearly independent, there would exist numbers $\gamma_{0}, \gamma_{1}, \ldots, \gamma_{m-1}$, not all zero such that

$$
\sum_{j=0}^{m-1} \gamma_{j} v_{j}(t)=0
$$

for all $t$. But $D^{k} v_{j}(0)=0, k \neq j, 0 \leqq k \leqq m-1, D^{j} v_{j}(0) \neq 0$, so $\gamma_{j}=0, j=0,1, \ldots, m-1$. This contradiction proves that the set $\left\{v_{j}\right\}_{j=0}^{m-1}$ is linearly independent and hence $\operatorname{dim} A^{+} \geqq m$.

The proof that, under the hypothesis of Lemma 3.1, $\operatorname{dim} A^{-} \geqq m$ follows easily from the inequality $\operatorname{dim} A^{+} \geqq m$ by means of a convenient artifice. For $k=0$, $1, \ldots, m$, define functions $\tilde{a}_{k}(t)=a_{k}(-t), t \in(-\infty, \infty)$. Clearly $\tilde{a}_{k} \in C^{k}(-\infty, \infty)$ and inf $\tilde{a}_{k}=\inf a_{k}=c_{k}$. Therefore, by what we have just shown, there exist $m$ linearly independent solutions $\tilde{v}_{0}, \tilde{v}_{1}, \ldots, \tilde{v}_{m-1}$ of the differential equation

$$
\sum_{k=0}^{m}(-1)^{k} D^{k}\left(\tilde{a}_{k} D^{k} y\right)=0
$$


such that $D^{k} \tilde{v}_{j} \in L^{2}[0, \infty)$ for $0 \leqq k \leqq m, 0 \leqq j \leqq m-1$. If for $j=0,1, \ldots, m-1$, $\omega_{j}(t)=\tilde{v}_{j}(-t)$, it is easy to verify that $\omega_{j}$ is a solution of

$$
\sum_{k=0}^{m}(-1)^{k} D^{k}\left(a_{k} D^{k} y\right)=0 .
$$

Therefore, since $D^{k} \omega_{j} \in L^{2}(-\infty, 0], 0 \leqq k \leqq m$, and the set $\left\{\omega_{j}\right\}_{j=0}^{m-1}$ is linearly independent, $\operatorname{dim} A^{-} \geqq m$.

The second assertion of Theorem 1 is a consequence of the following:

Lemma 3.3. Suppose in addition to the hypothesis of Lemma 3.1, $a_{k}$ is bounded above as well as below for $0 \leqq k \leqq m$. If $u$ is a solution of (1) such that $D^{k} u \in L^{2}(-\infty, \infty)$ for $0 \leqq k \leqq m$, then $u(t)=0$ for all $t \in(-\infty, \infty)$.

Proof. Referring to the proof of Lemma 2.3 we see that there exists a sequence of function $\left\{u_{n}\right\}_{n=1}^{\infty}$ such that

$$
u_{n}(t)=0 \quad \text { if }|t| \geqq n+1, \quad u_{n} \in C^{2 m}(-\infty, \infty),
$$

and

$$
\lim _{n \rightarrow \infty} D^{k} u_{n}=D^{k} u \quad \text { in } L^{2}(-\infty, \infty) \text { for } 0 \leqq k \leqq m .
$$

Since for $n=1,2, \ldots$

$$
\int_{-\infty}^{\infty} u_{n}(s) \sum_{k=0}^{m}(-1)^{k} D^{k}\left(a_{k} D^{k} u\right)(s) d s=0,
$$

it follows from (29) and integration by parts that

$$
\int_{-\infty}^{\infty} \sum_{k=0}^{m} a_{k}(s)\left(D^{k} u_{n}(s)\right)\left(D^{k} u(s)\right) d s=0 .
$$

By the boundedness of $a_{k}, 0 \leqq k \leqq m,(30)$ implies that

$$
\int_{-\infty}^{\infty} \sum_{k=0}^{m} a_{k}(s)\left(D^{k} u(s)\right)^{2} d s=0
$$

Let the numbers $d_{0}, d_{1}, \ldots, d_{m}$ and $\delta>0$ be defined as in the proof of Lemma 3.1. Since $\sum_{k=0}^{m} d_{k} \omega^{2 k} \geqq 0$, Lemma 2.3 implies that

Therefore

$$
\int_{-\infty}^{\infty} \sum_{k=0}^{m} d_{k}\left(D^{k} u(s)\right)^{2} d s \geqq 0 .
$$

$$
\begin{aligned}
\delta \sum_{k=0}^{m} \int_{-\infty}^{\infty}\left(D_{k} u(s)\right)^{2} d s & \leqq \delta \sum_{k=0}^{m} \int_{-\infty}^{\infty}\left(D_{k} u(s)\right)^{2} d s+\sum_{k=0}^{m} \int_{-\infty}^{\infty} d_{k}\left(D^{k} u(s)\right)^{2} d s \\
& =\sum_{k=0}^{m} \int_{-\infty}^{\infty} c_{k}\left(D^{k} u(s)\right)^{2} d s \leqq \sum_{k=0}^{m} \int_{-\infty}^{\infty} a_{k}(s)\left(D^{k} u(s)\right)^{2} d s=0
\end{aligned}
$$

and so $u(t)=0$ for all $t \in(-\infty, \infty)$. 
The second assertion of Theorem 1 now follows by a well known result in algebra. Assuming the hypothesis of Lemma 3.3 we have as an equivalent statement

Therefore

$$
\text { dimension } A^{+} \cap A^{-}=0 \text {. }
$$

$$
\text { dimension } A^{+}+\operatorname{dimension} A^{-} \leqq \operatorname{dimension} S=2 m
$$

(see for example $[2, \S 12$, problem $7(\mathrm{~b})]$ ). But we have shown that $\operatorname{dim} A^{+} \geqq m$, $\operatorname{dim} A^{-} \geqq m$; hence $\operatorname{dim} A^{+}=\operatorname{dim} A^{-}=m$.

The final statement of Theorem 1 is a consequence of the following elementary fact:

LEMMA 3.4. If $f \in C^{1}[0, \infty)$ and $f \in L^{2}[0, \infty), f^{\prime} \in L^{2}[0, \infty)$, then $\lim _{t \rightarrow \infty} f(t)=0$.

Proof. The hypothesis implies that $2 f f^{\prime} \in L^{1}[0, \infty)$. Therefore the identity $f(t)^{2}=f(0)^{2}+2 \int_{0}^{t} f(s) f^{\prime}(s) d s$ implies that $\lim _{t \rightarrow \infty} f(t)$ exists. But $f \in L^{2}[0, \infty)$ so $\lim _{t \rightarrow \infty} f(t)=0$.

This concludes the proof of Theorem 1.

4. Equations defined on a half-infinite interval-Examples. The following statement is actually a corollary of Theorem 1 :

THEOREM 2. Let $a_{k}, 0 \leqq k \leqq m$, be real functions defined on the half-infinite interval $[b, \infty)$ with $a_{k} \in C^{k}$. Assume each $a_{k}$ is bounded below and if $c_{k}=\inf a_{k}, 0 \leqq k \leqq m$, then $c_{m}>0$ and the polynomial (2) has no zero or purely imaginary roots. If $A$ denotes the vector space of real solutions of

$$
\sum_{k=0}^{m}(-1)^{k} D^{k}\left(a_{k} D^{k} y\right)=0
$$

which together with their first $m$ derivatives belong to $L^{2}[b, \infty)$, then $\operatorname{dim} A \geqq m$. If each $a_{k}$ is bounded above as well as below on $[b, \infty)$, then $\operatorname{dim} A=m$.

Proof. Let $\varphi$ be a real $C^{\infty}$ function defined on $(-\infty, \infty)$ such that

$$
\begin{aligned}
0 \leqq \varphi(t) & \leqq 1, & & t \in(-\infty, \infty), \\
\varphi(t) & =0, & & t \leqq b+1, \\
\varphi(t) & =1, & & t \geqq b+2 .
\end{aligned}
$$

For $k=0,1, \ldots, m$, define $a_{k}^{*} \in C^{k}(-\infty, \infty)$ by the formula

$$
a_{k}^{*}(t)=[1-\varphi(t)] c_{k}+\varphi(t) a_{k}(t) .
$$

Since for $k=0,1, \ldots, m$

$$
\inf _{(-\infty, \infty)} a_{k}^{*}=\inf _{[b, \infty)} a_{k}=c_{k},
$$

Theorem 1 implies that the differential equation

$$
\sum_{k=0}^{m}(-1)^{k} D^{k}\left(a_{k}^{*} D^{k} y\right)=0
$$


has $m$ linearly independent solutions which together with their first $m$ derivatives belong to $L^{2}[0, \infty)$. For $t \geqq b+2$ these solutions are also solutions of (1). Continuing these solutions back from $b+2$ to $b$ we obtain $m$ linearly independent solutions of (1) which are in $A$. This proves the first assertion of Theorem 2.

Suppose that each $a_{k}$ is bounded above as well as below on $[b, \infty)$ and contrary to the second assertion of Theorem $2, \operatorname{dim} A \geqq m+1$. This clearly implies that (1") has $m+1$ linearly independent solutions which together with their first $m$ derivatives belong to $L^{2}[0, \infty)$. But if each $a_{k}$ is bounded above on $[b, \infty)$ each $a_{k}^{*}$ is bounded above on $(-\infty, \infty)$ so we have a contradiction to Theorem 1 . This contradiction proves Theorem 2 .

We conclude with some simple but noteworthy examples:

1. Assume that both the first and second hypothesis of Theorem 1 and in addition that each $a_{k}$ is periodic with the same period $T>0$. It is known (see for example [1, Chapter 3]) that every solution of (1) can be expressed as a linear combination of solutions of the form

$$
e^{\lambda t} \sum_{j=0}^{r} p_{j}(t) t^{j}
$$

where $p_{j}(t+T)=p_{j}(t)$. The numbers $\lambda$ are the characteristic numbers of (1). If $y$ is a solution of (1) then $y \in A^{+}\left(y \in A^{-}\right)$if and only if in the linear combination of the solutions of the form (32) (comprising $y$ ) those solutions with $\operatorname{Re}(\lambda) \geqq 0(\operatorname{Re}(\lambda) \leqq 0)$ do not appear. Hence if $E^{+}\left(E^{-}\right)$denotes the subspace of solutions tending to zero exponentially as $t \rightarrow+\infty(t \rightarrow-\infty)$ it follows that $E^{+}=A^{+}, E^{-}=A^{-}$. Hence by Theorem 1,

$$
\begin{gathered}
\text { dimension } E^{+}=\text {dimension } E^{-}=m, \\
\text { dimension } E^{+} \cap E^{-}=0 .
\end{gathered}
$$

From the above discussion it also follows that if $y \in E^{+}\left(y \in E^{-}\right)$and $y$ is not identically zero then $y$ is unbounded on $(-\infty, 0]($ on $[0, \infty)$ ). Thus since (33) and (34) imply that every solution $y$ of (1) can be represented uniquely in the form $y=y_{1}+y_{2}, y_{1} \in E^{+}, y_{2} \in E^{-}$it follows that there exists no nontrivial solution of (1) bounded on $(-\infty, \infty)$. In particular, (1) has no periodic solution other than the trivial one.

2. Consider the fourth order selfadjoint differential equation

$$
\left(r y^{\prime \prime}\right)^{\prime \prime}+\left(q y^{\prime}\right)^{\prime}+p y=0 .
$$

If $r \in C^{2}[b, \infty), q \in C^{1}[b, \infty), p \in C[b, \infty)$, inf $r=R>0$, sup $q=Q<+\infty$, inf $p=P>0$, and either $Q<0$ or $Q^{2}-4 R P<0$, then by Theorem 2, there exist two independent solutions $u_{k}, k=1,2$, of (35) such that $u_{k}, u_{k}^{\prime} \in L^{2}[b, \infty), k=1,2$. For the special case $r(t)=1, q(t)=0$ for all $t \in[b, \infty)$, this result was discovered by Švec [3].

3. Finally consider the classical second order selfadjoint equation

$$
\left(r y^{\prime}\right)^{\prime}+q y=0
$$


where $r \in C^{1}[b, \infty), q \in C[b, \infty)$. If

$$
\sup r=R<0, \quad \inf q=Q>0,
$$

then by Theorem $2,(36)$ has a nontrivial solution $u$ such that $u, u^{\prime} \in L^{2}[b, \infty)$. It is easy to see that any other solution of (36) with this property must be of the form $\mathrm{cu}$. Indeed if $v$ is a solution with $v(b)>0, v^{\prime}(b)>0$ then since $d r v v^{\prime} / d t=r\left(v^{\prime}\right)^{2}-q v^{2}<0$, $v(t)>0, v^{\prime}(t)>0$ for all $t \in[b, \infty)$. Since $u$ and $v$ are independent solutions of (36) any other solution $y$ has the form $c_{1} u+c_{2} v$ and hence $y, y^{\prime} \in L^{2}[b, \infty)$ if and only if $c_{2}=0$. Thus dimension $A=1$ regardless of whether or not $r$ is bounded below and $q$ is bounded above.

\section{REFERENCES}

1. Earl A. Coddington and Norman Levinson, Theory of ordinary differential equations, McGraw-Hill, New York, 1955. MR 16, 1022.

2. Paul R. Halmos, Finite dimensional vector spaces, 2nd ed., The University Series in Undergraduate Math., Van Nostrand, Princeton, N. J., 1958. MR 19, 725.

3. M. Švec, Sur le comportement asymptotique des intégrals de l' équation différentielle $y^{(4)}+Q(x) y=0$, Czechoslovak Math. J. 8 (83) (1958), 230-245. MR 21 \#167.

\section{WhitMan COllege,}

Walla Walla, Washington 99362

CASE Western RESERVE UNIVERSITY,

Cleveland, Оhio 44106 